\title{
Scab Susceptibility of a Provenance Collection of Pecan in Three Different Seasons in the Southeastern United States
}

Clive H. Bock, United States Department of Agriculture - Agriculture Research Service, Southeastern Fruit and Tree Nut Research Lab (USDA-ARS, SEFTNRL), Byron, GA 31008; Larry J. Grauke, USDA-ARS Pecan Breeding and Genetics, Somerville, TX 77845; Patrick Conner, Horticulture Department, University of Georgia, Tifton, GA 31794; Susan L. Burrell, Hawkinsville High School, Hawkinsville, GA 31036; Michael W. Hotchkiss, USDA-ARS, SEFTNRL, Byron, GA 31008; Debbie Boykin, USDA-ARS, Stoneville, MS 38776; and Bruce W. Wood, USDA-ARS, SEFTNRL, Byron, GA 31008

\begin{abstract}
Bock, C. H., Grauke, L. J., Conner, P., Burrell, S. L., Hotchkiss, M. W., Boykin, D., and Wood, B. W. 2016. Scab susceptibility of a provenance collection of pecan in three different seasons in the southeastern United States. Plant Dis. 100:1937-1945.

Pecan scab (caused by Fusicladium effusum) is the most economically destructive disease of pecan in the Southeast United States. Wet, humid conditions typical of the Southeast are known to provide conditions conducive to epidemics. A provenance collection of pecan from 19 locations representing the native range of the tree is located in Byron, Georgia, and was assessed for pecan scab severity in 1998, 2013, and 2014. There were significant differences among the 19 provenances $(\mathrm{F}=5.6$ to $62.5, P<$ 0.0001 ). Provenances from wetter locations (generally north of Texas) had the greatest proportion of scab resistant trees, while provenances from the drier southern areas (Texas and Mexico) tended to be the most susceptible to scab. The association with rainfall was borne out by correlation analysis $(\mathrm{r}=-0.625$ to $-0.823[P<0.0001$ to 0.004$])$. Other factors consistently associated with scab severity included leaflet tilt and droop angle $(\mathrm{r}=-0.533$ to $-0.883[P<0.0001$ to 0.02$])$. Multiple regression

analysis demonstrated that leaflet droop angle was a particularly good predictor of provenance susceptibility. Leaflet characteristics vary with provenance location, and whether there is a direct relationship between scab severity and leaflet characteristics is not established. Estimates of heritability were not entirely consistent among years, but different methods were used to assess scab severity in 1998 (a 1 to 5 category scale) compared with 2013 and 2014 (the percent ratio scale). Despite using different methods, there was generally good agreement among years in regard to severity of disease on individual trees. In conclusion, trees from more northern populations (in areas with greater annual rainfall) are most likely to provide valuable and diverse sources of resistance to scab. The provenance collection contains a range of scab-resistant genotypes from diverse locations that can contribute to genetic improvement regarding scab resistance.
\end{abstract}

Pecan scab (caused by the plant pathogenic fungus Fusicladium effusum G. Winter) is the most destructive disease of pecan (Carya illinoinensis [Wangenh.] K. Koch) in the Southeast United States. Yield loss can be substantial (Gottwald and Bertrand 1982, 1988; Sanderlin 1995; Stevenson and Bertrand 2001), and much of the acreage in the Southeast is planted to susceptible and moderately susceptible cultivars. Not only does scab cause yield loss, but due to the stress it causes the tree, it can initiate cycles of alternate bearing with consequent production issues for growers. Scab epidemics are favored by warm, wet weather (Gottwald 1985; Gottwald and Bertrand 1982, 1983; Latham 1982), which is typical in the Southeast during the summer.

Several methods are used to manage scab, including application of fungicides, careful selection of orchard location and tree management (for example, thinning and hedge pruning), and the use of resistant cultivars. Unfortunately, F. effusum has now developed insensitivity to several classes of fungicide (Seyran et al. 2010; Stevenson 1999; Stevenson et al. 2004), and development of new chemistries is hampered by environmental sensitivity to use of pesticides for agriculture. Furthermore, scab can be particularly troublesome to control in the upper canopy of tall trees where spray equipment is unable to deliver sufficient fungicide (Bock et al. 2013, 2015). Orchard location, tree thinning, or hedge pruning can offer some measure of disease reduction, but are not solutions to the problem (Bock et al. 2014b; Cooper

Corresponding author: Clive H. Bock, E-mail: clive.bock@ars.usda.gov

*The $\boldsymbol{e}$-Xtra logo stands for "electronic extra" and indicates that three supplementary tables and five supplementary figures are available online.

Accepted for publication 29 March 2016.

http://dx.doi.org/10.1094/PDIS-12-15-1398-RE

This article is in the public domain and not copyrightable. It may be freely reprinted with customary crediting of the source. The American Phytopathological Society, 2016. and Johnson 1985). With many resistant cultivars that have been released, there is a history of resistance gradually declining over time (Cole and Gossard 1956; Goff et al. 1996), and the pathogen appears to adapt to the resistance presented by the host (Thompson and Grauke 1994). Indeed, the scab fungus is known to be genetically diverse throughout its range (Bock et al. 2014a, c), and there are several examples demonstrating pathogenic specialization of the pathogen to specific cultivars, or to a small range of generally related cultivars (Conner 2013; Conner and Stevenson 2004; Converse 1960; Demaree and Cole 1929). Nonetheless, in the long term, resistant cultivars are the single most desirable method for managing scab. However, there are many issues to resolve before a fully informed approach can be developed to optimize use of the range of available sources of resistance. Indeed, the number and diversity of resistance genes involved is yet to be characterized.

Breeding for scab resistance is a slow process, taking approximately 25 years from the initial cross to the time of release (Thompson and Grauke 1994). A tree of a resistant cultivar, once released, is continuously exposed to the pathogen population for decades or more, providing ample time for the fungus to adapt. Thus, it is desirable for any resistant cultivars developed to be based on a choice of resistance that will be durable. Currently there is no knowledge of the genetics of resistance as only limited crosses have been done, precluding the identification and tracking of specific resistance genes in any crosses. Nonetheless, some progress has been made in crossing various parental lines, which seems to indicate heritability of scab resistance is fairly high $\left(h^{2} \sim 0.50\right)$ (Thompson and Grauke 1994).

Although a linkage map has been developed for pecan (Beedanagari et al. 2005), and a few molecular markers have been developed (Conner and Wood 2001; Grauke et al. 2003a), these have only been used for cultivar identification and are not linked specifically to traits of interest for breeding purposes, such as scab resistance. This is unlike in apple, where linkage groups are becoming better characterized in relation to resistance genes in the host and virulence genes in the apple scab pathogen, Venturia inaequalis (for example, Erdin et al. 2006; Xu et al. 2009). 
A provenance collection of pecan representing the native geographic range of the species is planted in Byron, GA (and partially duplicated in Burleson and Brown counties, TX). The genetic diversity represented in the provenance collection is comparable to that of wild populations (Rüter et al. 1999). The collection has been described previously, and characteristics of the trees have been described in relation to source locations (Grauke et al. 1989, 2011; Rüter et al. 1999; Wood et al. 1998). However, the collection has not been assessed for resistance to pecan scab. The location in Byron, GA, has regular summer rainfall and frequent epidemics of scab. Characterizing the relationship between scab susceptibility and provenance may help provide insights into the locations where useful germplasm with novel sources of resistance to $F$. effusum may be found.

The objective of this study was to assess patterns of scab susceptibility in relation to the geographic range of pecan. Specifically, it was to identify those provenances and families within provenances that had greater resistance to scab, thereby indicating sources and locations of material of potential value to pecan breeding programs.

\section{Materials and Methods}

The provenance collection. The pecan provenance collection located at the USDA-ARS-SEFTNRL in Byron, GA $\left(32^{\circ} 39^{\prime} 54^{\prime \prime} \mathrm{N}\right.$, $83^{\circ} 44^{\prime} 31^{\prime \prime} \mathrm{W}$ ), has been described in detail previously (Grauke et al. 1989; Rüter et al. 1999; Wood et al. 1998). The site has an elevation of $\sim 156 \mathrm{~m}$ and $\sim 240$ days freeze-free growing period and annual precipitation of $\sim 118 \mathrm{~cm}$, and has Faceville sandy loam soils (FoA; fine, Kaolintic, thermic Typic Kandiudult soil). The provenances, their locations, physical characteristics, and some characteristics of the foliage are shown (Table 1). The trees represent collections of wild pecans from 19 provenances from locations in Missouri in the northern United States to Oaxaca in southern Mexico. As previously described in detail (Wood et al. 1998), nuts from five to six arbitrarily selected trees were sampled in each provenance. At each provenance location, intertree distance $(50 \mathrm{~m}$ to $10 \mathrm{~km}$ ) was maximized during the sampling process to reflect the genetic nature in that area. Nuts from each tree sampled within a provenance thus represented a "family" (further details of the collection and planting are found in Grauke et al. 1989, 2011; Wood et al. 1998).

Nuts from each of the five to six trees in each of the provenances were subsequently germinated in March 1989 (Wood et al. 1998), and were planted in the field. A completely randomized design was imposed to allow analysis of traits among provenances, with replicates (10) being represented by the seedlings from each of the five parent trees (families) within each of the 19 provenances $(n=\sim 960)$. Inter tree distance is $10.5 \mathrm{~m}$ within row and $10.5 \mathrm{~m}$ between rows. Orchard management is typical for pecans in Georgia (Wells et al. 2015), although no pesticides were applied.

Apart from source location and environmental conditions at source location, various other morphological parameters have been measured on these trees (Wood et al. 1998). Specifically included in this study were date of bud break (day of the year), leaflet droop angle, and leaflet tilt angle. Based on four leaves sampled in the lower canopy, Wood et al. (1998) measured droop angle as the angular distance between the abaxial planes of opposite leaflets at the center of a compound leaf (flat compound leaves have a large droop angle, while leaves that droop to form a "rib-cage" appearance have smaller droop angles). The leaflet tilt angle is the angular distance the plane of the base of leaflets project above the plane of the rachis of the compound leaf to which the leaflet is attached.

The orchard was transplanted from the original location to a second location at the USDA-ARS-SEFTNRL farm in March 2007. Approximately the same design was maintained and trees were replanted in a similar tree and replicate sequence as at the original site, although the row length and number of rows was different, and some trees were missing due to tree death, although tree survival after transplant was close to $100 \%$. A few of the trees were placed in different replicates at the new planting. If trees were not matched in each replicate with the original planting, they were not included in comparisons between years. Trees were trimmed back to the main trunk to reduce transplant shock and aid survival. Site characteristics and tree management were as described for the first planting site. Trees were drip-irrigated at both locations following standard management practice for pecans.

Weather conditions. Weather data were recorded close to the provenance planting using weather stations (Campbell Scientific, Logan, UT) and was downloaded from the Georgia Automated Environmental Monitoring Network (http://www.weather.uga.edu/). Mean temperature, relative humidity, and total rainfall were reported from 1 March to 31 July in 1998 (Fort Valley State University, Fort Valley, Peach County, GA), 2013 and 2014 (USDA-ARS-SEFTNRL, Byron, Peach County, GA). This period ensures covering those months during which leaves would be susceptible to infection.

Table 1. The latitude, elevation, and climatic conditions prevailing at sample sites of pecan provenances collected throughout the native range of the tree, and some foliar characteristics of the trees possibly associated with scab susceptibility ${ }^{y}$. Locations listed by latitude, from north to south

\begin{tabular}{|c|c|c|c|c|c|c|c|c|c|c|c|}
\hline Country & State & County & Code & Latitude & $\begin{array}{c}\text { Elevation } \\
(\mathbf{m})\end{array}$ & $\begin{array}{c}\text { Precipitation } \\
(\mathbf{m m}, \text { Mar-Jul })^{\mathrm{z}}\end{array}$ & $\begin{array}{c}\text { Max } \\
\operatorname{temp}\left({ }^{\circ} \mathrm{C}\right)\end{array}$ & $\begin{array}{c}\text { Min } \\
\text { temp }\left({ }^{\circ} \mathrm{C}\right)\end{array}$ & $\begin{array}{c}\text { Budbreak } \\
\text { (day of year) }\end{array}$ & $\begin{array}{c}\text { Leaflet } \\
\text { droop }\left({ }^{\circ}\right)\end{array}$ & $\begin{array}{l}\text { Leaflet } \\
\text { tilt }\left(^{\circ}\right)\end{array}$ \\
\hline U.S. & Missouri & Livingston & MO-L & 39.083 & 213 & 542.8 & 12.4 & -24.5 & 93 & 135 & 50 \\
\hline U.S. & Illinois & Jersey & IL-J & 39.000 & 192 & 488.2 & 11.4 & -24.5 & 93 & 142 & 53 \\
\hline U.S. & Missouri & Vernon & MO-V & 37.083 & 226 & 632.2 & 13.9 & -22.0 & 93 & 143 & 47 \\
\hline U.S. & Kentucky & Webster & KY-W & 37.050 & 226 & 523.0 & 13.8 & -22.0 & 94 & 139 & 50 \\
\hline U.S. & Kansas & Cowley & KS-C & 37.033 & 347 & 566.9 & 14.6 & -22.0 & 96 & 135 & 48 \\
\hline U.S. & Kansas & Cherokee & $\mathrm{KS}-\mathrm{K}$ & 37.000 & 274 & 604.3 & 14.3 & -22.0 & 94 & 138 & 46 \\
\hline U.S. & Tennessee & Lake & TN-L & 36.033 & 94 & 597.7 & 14.8 & -19.0 & 93 & 144 & 53 \\
\hline U.S. & Mississippi & Washington & MS-W & 33.883 & 39 & 545.8 & 17.4 & -10.5 & 94 & 134 & 49 \\
\hline U.S. & Texas & Bowie & TX-B & 33.550 & 119 & 440.9 & 17.1 & -10.5 & 90 & 148 & 52 \\
\hline U.S. & Texas & Tom Green & TX-T & 31.367 & 580 & 242.1 & 18.7 & -13.5 & 85 & 122 & 43 \\
\hline U.S. & Texas & Val Verde & $\mathrm{TX}-\mathrm{V}$ & 30.000 & 313 & 248.9 & 21 & -8.0 & 81 & 110 & 38 \\
\hline U.S. & Texas & Gonzales & TX-G & 29.500 & 95 & 395.7 & 19.8 & -8.0 & 77 & 135 & 45 \\
\hline U.S. & Texas & Kinney & TX-K & 29.233 & 227 & 282.2 & 21.9 & -8.0 & 80 & 117 & 39 \\
\hline U.S. & Texas & Zavala & TX-Z & 28.883 & 341 & 294.6 & 21 & -5.5 & 79 & 122 & 43 \\
\hline Mexico & Tamaulipas & Jaumave & MX-J & 23.367 & 884 & 327.0 & 24.4 & -2.5 & 71 & 125 & 38 \\
\hline Mexico & S.L.P & Santa Catarina & MX-C & 22.067 & 1219 & 195.1 & 17.9 & -2.5 & 82 & 125 & 39 \\
\hline Mexico & Hidalgo & Ixmiquilpan & MX-I & 20.483 & 1829 & 244.5 & 14.2 & 0.5 & 81 & 126 & 39 \\
\hline Mexico & Jalisco & Sayula & MX-S & 19.917 & 1372 & 288.3 & 19.1 & 3.0 & 80 & 121 & 43 \\
\hline Mexico & Oaxaca & Oaxaca & MX-O & 17.083 & 1737 & 425.5 & 20.6 & 3.0 & 78 & 113 & 43 \\
\hline
\end{tabular}

y Table and additional data from Wood et al. (1998).

${ }^{\mathrm{z}}$ Rainfall is based in the period from 1 March to 31 July, when foliage would be susceptible across the geographic range. 
Disease severity data. Disease severity was estimated differently in 1998, 2013, and 2014. In 1998, trees were approximately $7 \mathrm{~m}$ tall, and in 2013 and 2014, they were 10 to $12 \mathrm{~m}$ tall. Assessments were made from 1 to 31 July in 1998, 12 July to 10 August in 2013, and 10
June to 28 July in 2014, respectively. In 1998, a total of 954 trees in all 10 replicates were assessed; in 2013, only seven replicates were assessed, totaling 588 trees, and in 2014, eight replicates were assessed totaling 756 trees. Replicate size varied as some replicates
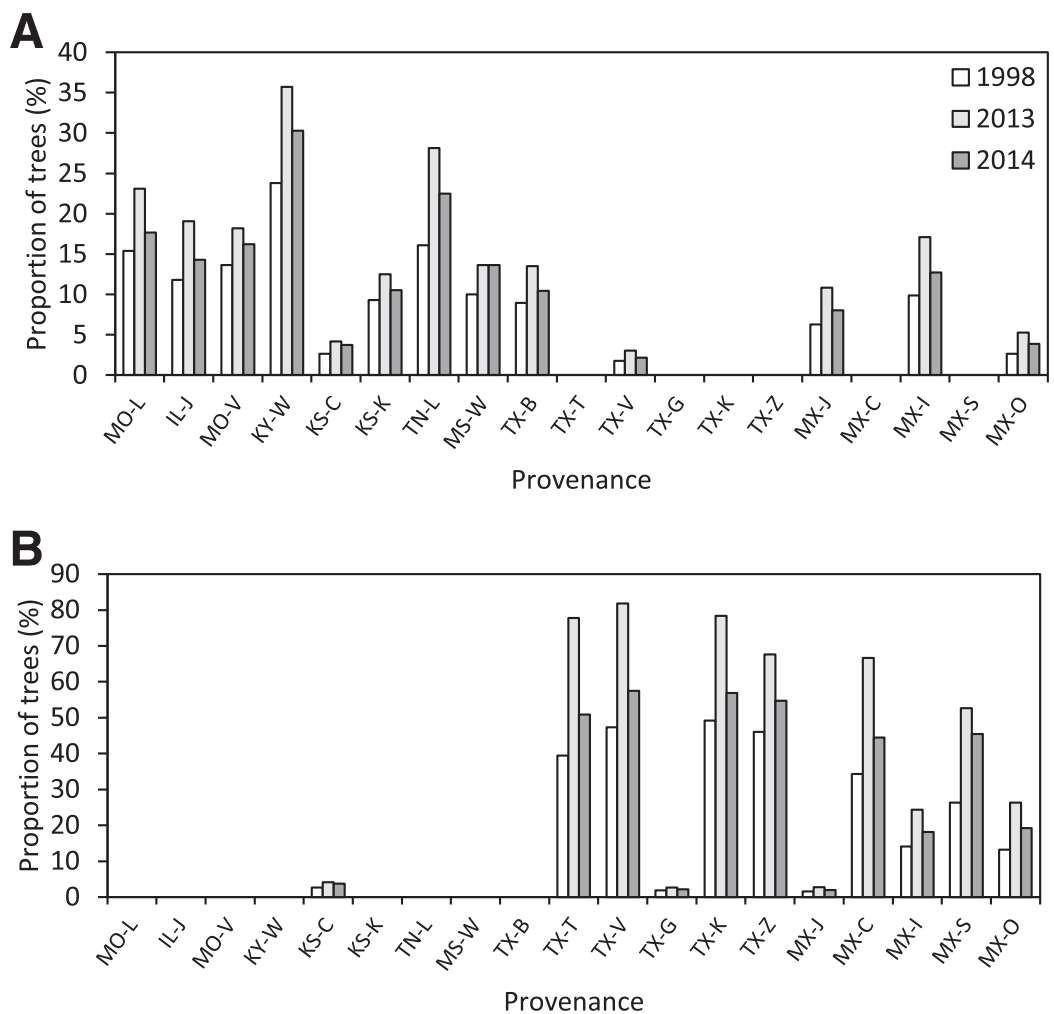

Fig. 1. The proportion of the total trees assessed each year in each provenance with consistent severity of pecan scab indicating $\mathbf{A}$, resistance (a rating score of 1 , or $\leq 0.1 \%$ leaf area diseased) or B, susceptibility (a rating score of 4 or 5 , or $\geq 0.5 \%$ leaf area diseased). The individual trees with these scored are presented in Supplementary Tables S2 and S3, respectively.

Table 2. Pecan scab severity by provenance in 1998, 2013, and 2014 at Byron, GA. In 1998, a 1 to 5 score was given to each plant based on an assessment of the scab on the single most severely infected leaf using the Hunter-Roberts scale $1=$ no infection, $2=$ one to several lesions restricted in size, $3=$ several large lesions on leaflets, $4=$ numerous leaflet and rachis lesions, some stem lesions may be present, and $5=$ leaflet and rachis lesions more numerous than above, stem lesions usually present. This was also converted to a percent severity based on the midpoint method. In 2013 and 2014, a sample of 10 leaves was taken from each plant and each was assessed for the $\%$ area with scab symptoms

\begin{tabular}{|c|c|c|c|c|}
\hline \multirow{2}{*}{$\begin{array}{l}\text { Provenance } \\
\text { (by latitude, } \mathbf{N} \rightarrow \mathbf{S} \text { ) }\end{array}$} & \multirow{2}{*}{$\frac{\text { Scab severity }(1 \text { to } 5)}{1998}$} & \multicolumn{3}{|c|}{ Scab severity $(\%)^{\mathrm{z}}$} \\
\hline & & 1998 & 2013 & 2014 \\
\hline MO-L & $1.737 \mathrm{def}$ & $0.500 \mathrm{f}$ & $0.067 \mathrm{de}$ & $0.145 \mathrm{~d}$ \\
\hline IL-J & $1.487 \mathrm{ef}$ & $0.162 \mathrm{f}$ & $0.081 \mathrm{de}$ & $0.166 \mathrm{~d}$ \\
\hline MO-V & $1.612 \mathrm{def}$ & $0.321 \mathrm{f}$ & $0.086 \mathrm{de}$ & $0.151 \mathrm{~d}$ \\
\hline KY-W & $1.587 \mathrm{def}$ & $0.221 \mathrm{f}$ & $0.033 \mathrm{e}$ & $0.202 \mathrm{~d}$ \\
\hline KS-C & 2.346 cdef & 1.873 ef & 0.172 cde & $0.199 \mathrm{~d}$ \\
\hline KS-K & $1.745 \mathrm{def}$ & $0.345 \mathrm{f}$ & $0.089 \mathrm{de}$ & $0.199 \mathrm{~d}$ \\
\hline TN-L & $1.359 \mathrm{f}$ & $0.141 \mathrm{f}$ & $0.012 \mathrm{e}$ & $0.146 \mathrm{~d}$ \\
\hline MS-W & $1.776 \mathrm{def}$ & $0.651 \mathrm{f}$ & $0.033 \mathrm{e}$ & $0.120 \mathrm{~d}$ \\
\hline TX-B & $1.562 \mathrm{def}$ & $0.218 \mathrm{f}$ & $0.008 \mathrm{e}$ & $0.191 \mathrm{~d}$ \\
\hline TX-T & $4.496 \mathrm{a}$ & $30.951 \mathrm{a}$ & $1.204 \mathrm{ab}$ & $0.200 \mathrm{~d}$ \\
\hline $\mathrm{TX}-\mathrm{V}$ & $4.291 \mathrm{a}$ & $23.774 \mathrm{ab}$ & $0.829 a b c$ & $0.390 \mathrm{bcd}$ \\
\hline TX-G & $2.602 \mathrm{~cd}$ & 2.066 ef & $0.073 \mathrm{de}$ & $0.250 \mathrm{~cd}$ \\
\hline TX-K & $4.324 \mathrm{a}$ & $24.251 \mathrm{ab}$ & $1.319 \mathrm{ab}$ & $1.009 \mathrm{abc}$ \\
\hline TX-Z & $4.102 \mathrm{a}$ & $19.984 \mathrm{bc}$ & $1.436 \mathrm{a}$ & $0.392 \mathrm{bcd}$ \\
\hline MX-J & $2.490 \mathrm{cde}$ & $1.953 \mathrm{f}$ & $0.019 \mathrm{e}$ & $0.197 \mathrm{~d}$ \\
\hline MX-C & $4.352 \mathrm{a}$ & $26.649 \mathrm{ab}$ & $0.719 \mathrm{abcd}$ & $1.312 \mathrm{ab}$ \\
\hline MX-I & $3.062 \mathrm{bc}$ & $8.864 \mathrm{~d}$ & 0.386 bcde & $0.538 \mathrm{abcd}$ \\
\hline MX-S & $4.017 \mathrm{ab}$ & $17.373 \mathrm{bcd}$ & 0.38 bcde & $1.613 \mathrm{a}$ \\
\hline MX-O & $3.409 \mathrm{abc}$ & $9.741 \mathrm{cde}$ & 0.416 abcde & $0.453 \mathrm{abcd}$ \\
\hline F-value ( $P$ value) & $30.7(<0.0001)$ & $62.5(<0.0001)$ & $11.4(<0.0001)$ & $5.6(<0.0001)$ \\
\hline
\end{tabular}

${ }^{\mathrm{z}}$ The $\%$ area diseased estimates were square root transformed. Back transformed values are presented with means separation for the transformed values. Means separation was based on Tukey's HSD $(\alpha=0.05)$. 
had more than one tree represented from each family. Complete replicate sets were collected and assessed in each year.

1998. In each tree, the leaf with the most severe scab was selected and severity was estimated based on the Hunter-Roberts category scale (Hunter and Roberts 1978), where $1=$ no infection, $2=$ one to several lesions restricted in size, $3=$ several large lesions on leaflets, $4=$ numerous leaflet and rachis lesions, some stem lesions may be present, and $5=$ leaflet and rachis lesions more numerous than above, stem lesions usually present. Raters placed the sample leaf in the category most appropriate to the severity of the symptoms. These category data were converted to a percent severity based on the midpoint method (Campbell and Madden 1990), by using the severity depicted on the guide leaves provided by Hunter and Roberts (1978) which was measured by image analysis (Lamari 2008).

2013 and 2014. Raters estimated severity visually based on the percent ratio scale, which is a continuous scale. Severity was estimated as the percent area diseased with scab symptoms on the whole leaf. Ten leaves were sampled arbitrarily per tree to represent the range of leaf ages on terminals. The raters used a standard area diagram series of scab symptoms on whole leaves to interpolate estimates as an aid for the assessments (C. H. Bock, unpublished data). Standard area diagrams are demonstrated to increase accuracy

Table 3. Pecan scab severity by family and provenance in 1998, 2013, and 2014 at Byron, GA. In 1998, a 1 to 5 score was given to each plant based on an assessment of the scab on the single most severely infected leaf using the Hunter-Roberts scale $1=$ no infection, $2=$ one to several lesions restricted in size, 3 = several large lesions on leaflets, $4=$ numerous leaflet and rachis lesions, some stem lesions may be present, and $5=$ leaflet and rachis lesions more numerous than above, stem lesions usually present. This was also converted to a percent severity based on the midpoint method (Campbell and Madden 1990). In 2013 and 2014, a sample of 10 leaves was taken from each plant and each was assessed for the \% area with scab symptoms

\begin{tabular}{|c|c|c|c|c|c|}
\hline \multirow{2}{*}{$\begin{array}{l}\text { Provenance } \\
\text { (by latitude, } \mathbf{N} \rightarrow \mathbf{S} \text { ) }\end{array}$} & \multirow[b]{2}{*}{ Family } & \multirow{2}{*}{$\begin{array}{c}\text { Scab severity (1 to 5) } \\
1998\end{array}$} & \multicolumn{3}{|c|}{ Scab severity $(\%)^{\mathrm{y}}$} \\
\hline & & & 1998 & 2013 & 2014 \\
\hline MO-L & 1 & $1.231 \mathrm{tu}$ & $0.033 \mathrm{rs}$ & 0.138 nopqrstvw & 0.093 uwyz(2)abe \\
\hline MO-L & 3 & 2.400 fghijklmnopqrstu & 1.776 klmnopqrs & 0.022 qrstvw & 0.242 jklmnoqrstuwyz(2)ac \\
\hline MO-L & 4 & 1.750 ijklmnopqrstu & 0.397 nopqrst & 0.079 nopqrstvw & 0.119 qrstuwyz(2)abde \\
\hline MO-L & 5 & 1.786 lmnopqrstu & 0.466 qrs & 0.053 nopqrstvw & 0.142 stuwyz(2)abe \\
\hline IL-J & 1 & 1.539 opqrstu & $0.191 \mathrm{qrs}$ & 0.081 nopqrstvw & 0.141 tuwyz(2)ae \\
\hline IL-J & 2 & 1.714 mnopqrstu & 0.343 qrs & 0.105 nopqrstvw & 0.167 stuwyz(2)ae \\
\hline IL-J & 3 & $1.385 \mathrm{rstu}$ & $0.120 \mathrm{qrs}$ & 0.059 opqrstvw & 0.161 stuwyz(2)ae \\
\hline IL-J & 4 & $1.250 \mathrm{tu}$ & $0.039 \mathrm{rsw}$ & 0.063 opqrstvw & 0.164 stuwyz(2)ae \\
\hline IL-J & 5 & 1.539 opqrstu & $0.201 \mathrm{qrs}$ & 0.100 nopqrstvw & 0.197 qrstuwyz(2)ade \\
\hline MO-V & 1 & 2.000 cdefghijklmnopqrstu & 0.749 ghijkmnopqrsx & 0.045 nopqrstvw & 0.116 nopqrstuwyz(2)abd \\
\hline MO-V & 2 & 1.600 mnopqrstu & $0.254 \mathrm{qrs}$ & 0.114 nopqrstvw & 0.162 qrstuwyz(2)abde \\
\hline MO-V & 3 & 1.800 klmnopqrstu & 0.495 pqrs & 0.053 opqrstvw & 0.175 qrstuwyz(2)ade \\
\hline MO-V & 4 & 1.546 nopqrstu & $0.246 \mathrm{qrs}$ & 0.171 mnopqrstvwx & 0.132 stuwyz(2)abe \\
\hline MO-V & 5 & 1.333 pqrstu & 0.069 qrs & 0.073 nopqrstvw & 0.166 qrstuwyz(2)abde \\
\hline KY-W & 1 & 1.000 uy & $0.000 \mathrm{rsw}$ & 0.043 opqrstvw & 0.098 uwyz(2)abe \\
\hline KY-W & 2 & 1.333 jklmnopqrstu & 0.069 mnopqrs & 0.003 rstvwx & 0.250 ijklmnoqrstuwyz(2)ac \\
\hline KY-W & 3 & 2.000 klmnopqrstu & 0.775 pqrs & 0.065 nopqrstvw & 0.137 stuwyz(2)abe \\
\hline KY-W & 4 & 1.500 mnopqrstu & 0.156 pqrs & 0.027 qrstvw & 0.144 stuwyz(2)abe \\
\hline KY-W & 5 & 2.000 ijklmnopqrstu & 0.665 opqrsu & 0.058 mnopqrstvwx & 0.493 cdefghijklmnoqrstuwyz \\
\hline KS-C & 1 & 1.750 ijklmnopqrstu & 0.351 nopqrst & 0.025 nopqrstvw & 0.121 noqrstuwyz $(2)$ abd \\
\hline KS-C & 2 & 3.500 abcdfghijklmnopqrstu & 5.449 abcdefghijkmnopqrs & 0.507 cdefghijklmnopqrstvw & 0.209 hijklmnoqrstuwyz(2)abc \\
\hline KS-C & 3 & 3.000 cdfghijklmno & 3.311 kmnopqrs & 0.342 hijklmnopqrstvwx & 0.312 hijklmnoqrstuwyz(2)c \\
\hline KS-C & 4 & 1.833 klmnopqrstu & 1.066 opqrs & 0.145 mnopqrstvwx & 0.140 qrstuwyz(2)abde \\
\hline KS-C & 5 & 2.182 jklmnopqrstu & 1.133 opqrs & 0.041 nopqrstvw & 0.221 noqrstuwyz(2)ad \\
\hline KS-K & 1 & 1.167 opqrstu & 0.017 pqrs & 0.103 nopqrstvw & 0.205 noqrstuwyz( 2$) \mathrm{ad}$ \\
\hline $\mathrm{KS}-\mathrm{K}$ & 3 & 1.714 klmnopqrstu & 0.416 opqrs & 0.020 qrstvw & 0.121 stuwyz(2)abe \\
\hline KS-K & 4 & 2.333 jklmnopqrsty & 1.275 opqrs & 0.155 nopqrstvw & 0.263 noqrstuwy $(2) \mathrm{d}$ \\
\hline KS-K & 5 & 1.546 nopqrstu & $0.197 \mathrm{qrs}$ & 0.098 nopqrstvw & 0.210 qrstuwyz(2)ade \\
\hline TN-L & 1 & $1.111 \mathrm{u}$ & $0.007 \mathrm{~s}$ & $0.003 \mathrm{vw}$ & 0.119 uwyz(2)abe \\
\hline TN-L & 3 & $1.200 \mathrm{tu}$ & 0.024 qrs & $0.002 \mathrm{vw}$ & 0.092 wyz(2)ab \\
\hline TN-L & 4 & 1.462 pqrstu & $0.150 \mathrm{qrs}$ & 0.020 qrstvw & 0.090 wxyz(2)ab \\
\hline TN-L & 5 & 1.556 mnopqrstu & 0.207 qrsv & 0.085 nopqrstvw & 0.114 tuwyz(2)abe \\
\hline TN-L & 6 & 2.000 abcdfghijklmnopqrstu & 0.625 abcdefghijkmnopqrs & 0.001 nopqrstvw & 0.535 abcdefghijklmnoqrstuwyz(2)ab \\
\hline MS-W & 1 & 3.000 abcdfghijklmnopqrstu & 3.000 abcdefghijkmnopqrs & z & - \\
\hline MS-W & 2 & $1.429 \mathrm{qrstu}$ & $0.114 \mathrm{qrs}$ & 0.038 nopqrstvw & 0.100 qrstuwyz(2)abde \\
\hline MS-W & 3 & 2.600 dfghijklmnopqrstu & $2.068 \mathrm{jkmnopqrs}$ & 0.057 nopqrstvw & 0.149 noqrstuwyz( 2 )abd \\
\hline MS-W & 4 & 1.667 klmnopqrstu & 0.277 opqrs & $0.008 \mathrm{rstvw}$ & 0.157 qrstuwyz(2)abde \\
\hline MS-W & 5 & 1.000 mnopqrstux & 0.000 nopqrs & 0.043 nopqrstvw & 0.074 qrstuwyz(2)abde \\
\hline TX-B & 1 & 2.000 klmnopqrstu & 0.686 pqrs & $0.007 \mathrm{stvw}$ & 0.233 noqrstuwyz $(2) \mathrm{ad}$ \\
\hline TX-B & 2 & 1.455 opqrstu & 0.139 qrs & 0.043 pqrstvw & 0.173 qrstuwyz(2)ade \\
\hline TX-B & 3 & 1.667 lmnopqrstuw & 0.347 pqrs & 0.011 rstvw & 0.274 klmnoqrstuwyz \\
\hline TX-B & 4 & $1.273 \mathrm{tu}$ & 0.046 qrs & 0.002 tvwy & 0.157 qrstuwyz(2)abde \\
\hline TX-B & 5 & 1.417 pqrstu & $0.108 \mathrm{qrs}$ & $0.000 \mathrm{w}$ & 0.133 tuwyz(2)abe \\
\hline TX-T & 1 & 4.083 abcdfg & 19.878 abcdefghijkmt & 0.307 inopqrstvwx & $0.000(2) \mathrm{ab}$ \\
\hline TX-T & 2 & 4.667 abe & $39.172 \mathrm{abc}$ & 1.763 bcde & 0.408 fghijklmnoqrstuw \\
\hline TX-T & 3 & 4.636 abe & 34.113 abcdefg & $1.691 \mathrm{bcdefg}$ & 0.415 fghijklmnoqrstuw \\
\hline
\end{tabular}

y The $\%$ area diseased estimates were square root transformed. Back transformed values are presented with means separation for the transformed values. Mean separation was based on Tukey's HSD $(\alpha=0.05)$. Where ( 2$)$ is indicated, the means separation spans a second run of the alphabet for that means comparison.

z Missing values. 
of disease estimates (Yadav et al. 2013). Scab severity on leaves is generally closely related to that on fruit (Nutter et al. 1985), so is an appropriate measure of scab susceptibility to compare germplasm (Goff et al. 2003; Nesbitt et al. 1996; Sanderlin et al. 1981).

Statistical analysis. SAS V9.3 (SAS Institute, Cary, NC) was used for all the statistical analyses.

Differences among provenances and families. All scab severity data (1998 scale and converted \% area data, and 2013 and 2014 $\%$ area severity data) were analyzed using a general linear mixed model. To compare provenance means, a general linear mixed model was used with fixed effects of provenance and random effects of family nested in provenance and replicate nested in provenance (as families within provenances were not necessarily equally susceptible, and the five families are not the same for each provenance). To compare family means within provenance, the same general linear mixed model was used, but with fixed effects of both provenance and family nested in provenance. The Akaike (and Aikaike improved) and
Bayesian information criteria were checked during model selection in relation to random effects. Type III fixed effects were checked to confirm significance. Almost all \% severity estimates were $<30 \%$, and variance was proportional to the mean, so data were square root transformed to improve normality and homoscedasticity prior to analysis. Residuals were inspected, and results of the analysis were back-transformed for presentation. Category scale data were not transformed. A pairwise comparison of genotypes was made using Tukey-Kramer adjusted pairwise comparisons and was converted to letter groupings using an SAS macro (PDMIX800), which takes the probability values for differences among means and converts the information to letter groups, where means with a common letter are not statistically different at $\alpha=0.05$ (Saxton 1998).

Correlation analysis was used to compare severity of disease among families (averaged across replicate trees with each family within a provenance) and provenances (averaged across families within each provenance) in the different years; where category scale

Table 3. (continued from preceding page)

\begin{tabular}{|c|c|c|c|c|c|}
\hline \multirow{2}{*}{$\begin{array}{l}\text { Provenance } \\
\text { (by latitude, } \mathbf{N} \rightarrow \mathbf{S} \text { ) }\end{array}$} & \multirow[b]{2}{*}{ Family } & \multirow{2}{*}{$\begin{array}{c}\text { Scab severity (1 to } 5) \\
1998\end{array}$} & \multicolumn{3}{|c|}{ Scab severity $(\%)^{y}$} \\
\hline & & & 1998 & 2013 & 2014 \\
\hline TX-T & 4 & 4.588 abe & 31.595 abcdeg & 1.544 bcdefg & 0.612 efghijklmnoqrstuv \\
\hline TX-T & 5 & 4.500 abe & 31.789 abcdeg & 0.974 bcdefghiklm & $0.039 \mathrm{yz}(2) \mathrm{ab}$ \\
\hline TX-V & 1 & 3.778 abcdfghi & 7.124 hijkmnopqrs & 0.532 cdefghijklmnopqr & $0.016(2) \mathrm{b}$ \\
\hline TX-V & 2 & 3.846 abcdfgh & 17.104 bcdefghijkmnu & 0.486 fjklmnopqx & 0.259 otuwy(2)de \\
\hline $\mathrm{TX}-\mathrm{V}$ & 3 & 4.444 abcd & 24.650 abcdefghijk & 1.336 bcdeghi & 1.171 abcdefghi \\
\hline TX-V & 4 & $4.833 \mathrm{abc}$ & $45.420 \mathrm{abcd}$ & 0.782 cdefghijklmn & 0.604 cdefghijklmnoqrstuw \\
\hline TX-V & 5 & 4.643 abe & 34.460 abcdeg & 1.227 bcdefghij & 0.744 cdefghijklmnqrs \\
\hline TX-G & 1 & 3.000 abcdfghijklmnopqrstu & 3.000 bcdefghijkmnopqrs & 0.107 klmnopqrstvwx & 0.184 jklmnoqrstuwyz(2)abc \\
\hline TX-G & 2 & 2.750 ghijklmnopqr & 2.441 nopqrs & 0.034 qrstvw & 0.290 jklmnoqrstuwy(2)c \\
\hline TX-G & 3 & 2.182 jklmnopqrstu & 0.978 opqrs & 0.041 qrstvw & 0.193 qrstuwyz(2)ade \\
\hline TX-G & 4 & 2.571 hijklmnopqrst & 1.896 opqrs & 0.129 nopqrstvw & 0.288 mnoqrstuwy(2)d \\
\hline TX-G & 5 & 2.733 ghijklmnopq & 2.329 opqrs & 0.085 nopqrstvw & 0.297 klmnoqrstuwy \\
\hline TX-K & 1 & 4.000 abcdfgv & 12.584 cdefghijkmnop & 1.267 bcdefghij & 0.709 dfghijklmnoqrstv \\
\hline TX-K & 3 & $4.333 \mathrm{abcd}$ & 22.446 abcdefghijk & $1.682 \mathrm{bcd}$ & 0.613 dfghijklmnoqrstuvx \\
\hline TX-K & 4 & $4.733 \mathrm{ab}$ & $39.478 \mathrm{ab}$ & $2.274 \mathrm{ab}$ & $1.938 \mathrm{ab}$ \\
\hline TX-K & 5 & $4.444 \mathrm{abcd}$ & 28.495 abcdefghi & 0.541 efghijklmnopqru & 1.590 abce \\
\hline TX-K & 6 & $4.083 \mathrm{abcdfg}$ & 22.244 abcdefghijk & 1.058 cdefghijkl & 0.541 fghijklmnoqrstuw \\
\hline TX-Z & 1 & 4.308 abcd & 23.596 abcdefghijl & $1.610 \mathrm{bce}$ & 0.285 lstuwy $(2) \mathrm{d}$ \\
\hline TX-Z & 2 & $4.900 \mathrm{a}$ & $49.613 \mathrm{a}$ & $4.164 \mathrm{a}$ & 0.663 cdefghijklmnoqrstuw \\
\hline TX-Z & 3 & 3.556 abcdfghij & 10.092 defghijkmnopqw & 0.681 dfghijklmnou & $0.000 \mathrm{z}(2) \mathrm{ab}$ \\
\hline TX-Z & 4 & 3.583 abcdfghij & 9.099 fhijkmnopqx & 0.455 ghijklmnopqrsxy & 0.852 bcdefghijkmnoqr \\
\hline TX-Z & 5 & 4.167 abcdfgh & 18.122 abcdefghijkmno & 1.566 bcdef & 0.710 cdefghijklmnoqrstu \\
\hline MX-J & 1 & 2.000 klmnopqrstu & 0.720 pqrs & 0.004 tvw & 0.197 qrstuwyz(2)ade \\
\hline MX-J & 2 & 2.455 hijklmnopqrstu & 1.772 opqrs & 0.061 nopqrstvw & 0.126 tuwyz(2)abe \\
\hline MX-J & 3 & 2.833 fghijklmnop & 2.621 nopqrs & 0.046 nopqrstvw & 0.205 noqrstuwyz(2)ad \\
\hline MX-J & 4 & 2.929 fghijklmn & 3.027 nopqrs & 0.032 qrstvw & 0.315 jklmnoqrstuwy(2)c \\
\hline MX-J & 5 & 2.231 jklmnopqrstu & 2.098 opqrs & $0.000 \mathrm{w}$ & 0.169 stuwyz $(2) \mathrm{ae}$ \\
\hline MX-C & 1 & 4.100 abcdfg & 19.966 abcdefghijkmn & 0.815 bcdefghijklmnop & 1.197 abcdefghijklm \\
\hline MX-C & 2 & $4.600 \mathrm{abc}$ & 36.127 abcdeg & 0.796 bcdefghijklmno & 1.169 abcdefghijklm \\
\hline MX-C & 3 & 4.000 abcdfghijklmnopqrs & 21.307 abcdefghijkmnopqrs & 0.487 cdefghijklmnopqrstvw & 1.579 abcdefghi \\
\hline $\mathrm{MX}-\mathrm{C}$ & 4 & $4.500 \mathrm{abcd}$ & 30.850 abcdefgh & 0.796 cdefghijklmn & 1.323 abcdefgh \\
\hline MX-I & 1 & $1.375 \mathrm{stu}$ & $0.099 \mathrm{rs}$ & 0.130 nopqrstvw & 0.195 rwyz(2)ade \\
\hline MX-I & 2 & $4.267 \mathrm{abcd}$ & 28.284 abcdefgh & 1.127 bcdefghijk & 1.025 abcdefhijp \\
\hline MX-I & 3 & 3.429 bcdfghij & 11.243 efghijkmnopv & 0.482 efghijklmnopqru & 0.633 cdefghijklmnoqstu \\
\hline MX-I & 4 & 3.917 abcdfgh & 21.423 abcdefghijkm & 0.232 lmnopqrstvwx & 0.628 cdefghijklmnoqrstuw \\
\hline MX-I & 5 & 2.385 ijklmnopqrstu & 1.616 opqrs & 0.257 lmnopqrstvwx & 0.401 gklmnoqrstuwy(2)c \\
\hline MX-S & 1 & 3.429 abcdfghijkw & 5.225 hijkmnopqrs & 0.280 hijklmnopqrstvwx & $2.582 \mathrm{a}$ \\
\hline MX-S & 2 & 4.000 abcdfghi & 8.624 cdefghijkmnopqrs & 0.455 cdefghijklmnopqrstv & 1.694 abcde \\
\hline MX-S & 3 & 4.800 abcd & 43.393 abcdef & 0.169 lmnopqrstvwx & 1.209 bcdefghijkl \\
\hline MX-S & 4 & 4.167 abcdfgh & 30.134 abcdefghij & 0.409 cdefghijklmnopqrstvw & 1.142 bcdefghijklmno \\
\hline MX-S & 5 & 3.750 abcdfghijkl & 12.539 abcdefghijkmnopqrs & 0.734 bcdefghijklmnopqrst & 1.754 abcdefg \\
\hline MX-O & 1 & 3.333 abcdfghijklm & 11.627 bcdefghijkmnopqr & 0.792 bcdefghijklmnopq & 0.151 noqrstuwyz(2)abd \\
\hline MX-O & 2 & 2.546 hijklmnopqrstvy & 2.872 nopqrst & 0.112 mnopqrstvwx & 0.077 uwyz(2)abe \\
\hline MX-O & 3 & 3.333 abcdfghijkx & 4.805 ijkmnopqrs & 0.217 jklmnopqrstvwx & 0.239 jklmnoqrstuwyz(2)ac \\
\hline MX-O & 4 & 4.000 abcdfghijklmnopqrstu & 8.624 abcdefghijkmnopqrs & 1.103 abcdefghijklmnopqrs & $2.736 \mathrm{abcd}$ \\
\hline $\mathrm{MX}-\mathrm{O}$ & 5 & $4.429 \mathrm{abcdf}$ & 28.860 abcdefghij & 0.383 bcdefghijklmnopqrstvw & 0.543 bcdefghijklmnoqrstuwyz \\
\hline F-value ( $P$ value) & & $3.97(<0.0001)$ & $3.96(<0.0001)$ & $6.50(<0.0001)$ & $10.89(<0.0001)$ \\
\hline
\end{tabular}


data were used, Spearman's rank correlation was applied, but for continuous data, Pearson's correlation was used. Due to non-independence of data, associations of individual tree assessments between years were not subject to correlation analysis. In addition, correlation analysis was used to relate provenance scab severity to latitude, elevation, precipitation, temperature, budbreak date, leaflet droop angle, and leaflet tilt angle. $P$-values were inspected to ascertain associations between variables. A stepwise multiple regression analysis was applied to determine those physical factors (latitude, rainfall, mean maximum and mean minimum temperatures, and elevation) and those foliar characteristics (budbreak date, leaflet droop angle, and leaflet tilt angle) contributing most as predictors of scab severity among the different provenances (averaged across families within each provenance). An unweighted paired grouping of mathematical averages (UPGMA) cluster analysis was performed to assess the relationship among the various provenances based on scab severity data in each year.

Heritability. Heritability (Holland et al. 1998; Wright 1976) was calculated using a mixed model based on the same general linear mixed model described above, but with all sources modeled as random effects. Random effects of replicate and replicate $\times$ provenance were estimated to be zero and were removed from the model to simplify the analysis. Thus a reduced model based solely on provenance and family within provenance was used. The variance components of the model were obtained and used to estimate heritability as described in Holland et al. (1998) and on the website http://www4.ncsu.edu/ $\sim$ jholland/heritability/OneEnvironRCBDHeritability.sas. Heritability for both individual trees and families was calculated. As previously described (Wood et al. 1998), trees in the same family were assumed to be half-sibs as selfing in maternal trees was unlikely due to broad outcrossing. Each tree genotype was tested at only a single site (Byron, GA), precluding a separation of genotype-environment interactions from genetic variation among families and individuals, which thus slightly overestimates heritability (Wright 1976).

\section{Results}

Weather. Although mean maximum $\left(27.9,25.8\right.$, and $\left.27.2^{\circ} \mathrm{C}\right)$, mean minimum $\left(14.8,14.0\right.$, and $\left.14.4^{\circ} \mathrm{C}\right)$, and mean temperatures $\left(21.1,19.4\right.$, and $\left.20.4^{\circ} \mathrm{C}\right)$ were fairly similar during the period recorded in 1998, 2013, and 2014, respectively, rainfall was markedly different $(288.3,860.3$, and $549.4 \mathrm{~mm}$, respectively). In 1998 , there were relatively dry conditions during the growing season, but in 2013, conditions were particularly wet, and in 2014 they were moderately wet.

Estimates of severity. Despite different assessment methods used in 1998 ( 1 to 5 scale), and in 2013 and 2014 (the percentage ratio scale), there was a clear relationship between methods of assessment (Supplementary Fig. S1A to C). However, the frequency of severities in 1998, 2013, and 2014 showed different trends (Supplementary Fig. S2). Estimates based on the category scale were spread relatively evenly across the five categories. However, estimates in 2013 and 2014 indicated a preponderance of very low severities in both years $(<0.5 \%$ leaf area). The frequency of trees that had extreme susceptibility in 2013 and 2014 (>2\% leaf area) was relatively low. There was general agreement among severity estimates on individual trees from all years (Supplementary Fig. S3), although there were a few outliers between 2013 and 2014, and in 1998 trees scored as a 4 or a 5 (susceptible) did not necessarily have much leaf area diseased in 2013 and 2014. Assessment on individual trees among years shows the range of rating score in 1998 and of estimates of \% area in 2013 and 2014 (Supplementary Table S1). Thus, comparing only the trees that were common to all three years, in 1998, there were 165 trees that had a score of 1 (no scab) and 101 with a score of 5. However, in 2013 , there were 117 trees with a severity of $<0.2 \%$, and only 3 with a severity (\% leaf area) $>12.5 \%$, while in 2014 there were 134 trees with a severity of $<0.2 \%$, and only 4 with a severity (\% leaf area) $>12.5 \%$. In most cases, trees had comparable severity among years. Examples of individual trees that showed inconsistent results between years are: \#284 (IL-J-2), which was scored as 1, 2.1\%, and $0.1 \%$ in 1998, 2013, and 2014, respectively; \#364 (TX-V-3), which was scored as 5, 4.7\%, and $0.7 \%$ in 1998, 2013, and 2014, respectively; \#448 (TX-T-3), which was scored as 5, 11.4\%, and $0.9 \%$ in 1998, 2013, and 2014, respectively; and \#616 (TX-B-2), which was scored as $1,2.4 \%$, and $0.1 \%$ in 1998,2013 , and 2014 , respectively. An example of a tree with consistent severity is \#118 (MO-L-5), which was scored as $1,0.0 \%$, and $0.1 \%$ in 1998,2013 , and 2014, respectively. Indeed, the majority of trees were highly consistent in regard to severity rating. Thus, there were 66 trees that were consistently highly resistant across years and had a rating score of 1 or $\leq 0.1 \%$ area diseased (Supplementary Table S2). Similarly, there were 147 trees consistently scored as highly susceptible across years and had a severity score of 4 or 5 or $\geq 0.5 \%$ leaf area diseased (Supplementary Table S3). The consistency among these scores in regard to source provenance is demonstrated when considering these trees as a proportion of total trees assessed in each provenance in each year (Fig. 1). The greatest proportion of scab resistant trees were consistently found in provenances MO-L, IL-J, MO-V, KY-W, KS-K, TN-L, MS-W (more northerly provenances with higher rainfall), and TX-B, and to a lesser extent MX-J and MX-I. Conversely, the greatest proportion of scab susceptible trees were consistently found in provenances TX-T, TX-V, TX-K, TX-Z, MX-C, MX-S, and MX-O, and to a lesser extent MX-I, all drier, more southerly provenances.

Provenance and family scab resistance. In all three years, the severity of scab depended on provenance $(\mathrm{F}=5.6$ to $62.5, P<0.0001$, Table 2). In 1998, the severity score ranged from 1.359 (TN-L) to 4.496 (TX-T), in 2013 the mean leaf area diseased ranged from $0.008 \%$ (TX-B) to $1.436 \%$ (TX-Z), and in 2014 from $0.120 \%$ (MS-W) to $1.613 \%(\mathrm{MX}-\mathrm{S})$. Although there were some differences among ranking with provenances among years, nine provenances consistently had the most resistant genotypes in all years (TN-L, IL-J, TX-B, KY-W, KS-K, KS-C, MO-L, MO-V, and MS-W), while two provenances, MX-C and MX-O (when based on the scale in 1998) were consistently in the most susceptible group; TX-T, TX-V, TX-Z, MX-C, and MX-S were also grouped together with TX-K, and MX-O in 1998. Other provenances had intermediate scab severity. When ranking changed for a provenance between years, the change was never to extreme susceptibility or resistance. The UPGMA showed that certain provenances consistently clustered together (Supplementary Fig. S4). In all three seasons, there were two groupings, one comprising provenances with more scab susceptible trees (TX-T, TX-V, TX-K, and TX-Z, rainfall March to July $=242.1$ to $294.6 \mathrm{~mm}$ ), and the other with provenances having trees that tended to be more resistant (MO-L, MS-W, IL-J, KY-W, TX-B, TN-L, MO-V, KS-K, KS-C, MX-J, and TX-G, rainfall March to July $=327.0$ to $632.2 \mathrm{~mm}$ ).

Among families nested within provenance, there was also a range of susceptibilities in all three years $(\mathrm{F}=3.96$ to $10.89, P<0.0001$, Table 3). The means separation was similar among years, although ranking differed slightly, and the severities followed a similar pattern to that described for provenances. One of the provenances with the most resistant families was TN-L. In 1998, the severity scores ranged from 1.111 to 2.000 , and the severity estimates in 2013 and 2014 were 0.001 to 0.085 and 0.090 to 0.535 , respectively. For a more susceptible provenance, for example, TX-K, the severity scores for families in 1998 ranged from 4.000 to 4.733 , and the severity estimates in 2013 and 2014 were 0.541 to $2.274 \%$ and 0.541 to $1.938 \%$, respectively. The frequency of trees with different severities within provenances had a similar pattern among years (Supplementary Fig. S5), with those more susceptible provenances consistently having greatest frequency of trees with a high scab severity, and the more resistant provenances consistently having a greater frequency of trees with less severe scab. There was consistent correlation among provenances between years $(\mathrm{r}=0.891$ to $0.955, P<0.0001)$, and between families within provenances ( $\mathrm{r}=0.821$ to $0.896, P<0.0001$ ).

Provenance and severity of pecan scab in relation to environmental and host variables. In 1998, severity of pecan scab was negatively correlated with provenance source latitude $(\mathrm{r}=-0.693[P=$ 0.001], Table 4), but there was no association with latitude in 2013 or 2014. However, scab severity was negatively associated with precipitation at the provenance source location in all three seasons $(\mathrm{r}=$ -0.625 to $-0.823[P<0.0001$ to 0.004$])$, indicating that trees tended to be more resistant from locations where rainfall was higher. Scab 
severity was positively correlated with maximum temperature at the source location in all three seasons $(r=0.491$ to $0.677[P=0.002$ to $0.03]$ ), but with minimum temperature only in 1998. Similarly, provenance source elevation was positively associated with scab severity only in 1998. Severity was negatively correlated with budbreak in 1998 , but not in 2013 or 2014 . Scab severity was negatively correlated with both leaflet droop ( $\mathrm{r}=-0.700$ to $-0.883[P<0.0001$ to $0.0004])$ and leaflet tilt ( $\mathrm{r}=-0.533$ to $-0.844[P<0.0001$ to 0.02$])$ in all three years. The multiple regression analysis indicated precipitation and leaflet droop were good predictors of provenance scab susceptibility (Table 5).

Tree and family heritability of pecan scab resistance. Estimates of heritability varied among years and to a lesser degree assessment method (Table 6). Whereas family heritability was comparatively high when based on the 1 to 5 category scale in 1998, they were relatively low in 2013 and 2014 when based on the 0 to $100 \%$ scale. However, converting the percent scale data to the category scale improved the heritability estimates of scab resistance for 2013 and 2014. Single tree heritability was consistently lower in all years, and followed a similar pattern in relation to assessment method to that for family heritability.

\section{Discussion}

There was a substantial range in scab susceptibility in the pecan provenance collection. The collection is known to be genetically diverse (Grauke et al. 1995, 2003b; Rüter et al. 1999; Sagaram et al. 2011; Wood et al. 1998), and the reaction of trees from the different provenances to naturally developing epidemics of pecan scab at this location in Byron, GA, confirms this genetic diversity. The environmental conditions experienced at the source locations by these trees will most likely affect the frequency of specific alleles associated with traits such as scab susceptibility. In areas where scab pressure is high, the trees experience selection to develop and maintain scab resistance. This is particularly true when considering the toll that scab can take on reproductive capacity of a tree (Gottwald and Bertrand 1982, 1988; Sanderlin 1995; Stevenson and Bertrand 2001). Highly susceptible trees might not produce any offspring in severe scab epidemic seasons. However, where scab is not an issue, selection for scab resistance would be unlikely to be sufficient for these traits to become predominant in the pecan population.

Rainfall at the provenance location was consistently correlated with the severity of scab. Rainfall is known to be the driving force behind epidemics (Gottwald and Bertrand 1982, 1983; Latham 1982), and it is those provenances with the least rainfall that tended to have the more scab susceptible trees (TX-Z, TX-T, TX-V, TX-K, MX-O, MX-I, MX-S, and MX-C). Grauke et al. (1995) commented that in Arkansas, the climatic conditions in early spring are generally conducive to scab epidemics, and thus trees in that area may have adapted by developing resistance to the pathogen. The results of the current study bear out this observation-locations with higher rainfall tend to have a preponderance of trees that are more resistant to scab. Trees in these areas provide a valuable resource for resistance to scab. Indeed, it is material from these areas that have been utilized by the USDA in the scab resistance screening program (Thompson and Grauke 1991). Elevation has been shown to affect severity (Sparks et al. 2009), although only in 1998 was elevation found to be associated with scab severity in the current study. Similarly, temperature was associated with scab severity in 1998, but budbreak date, and in particular leaflet droop angle and leaflet tilt angle, were consistently negatively associated with the severity of scab among provenances. Both leaflet droop angle and leaflet tilt angle are positively correlated with latitude and rainfall (Wood et al. 1998), and thus the association between scab and leaflet characteristics may be spurious and due to other co-related factors, rather than leaflet characteristics directly affecting scab severity per se.

Within each provenance, trees from the different families exhibited a range of severity, demonstrating the diversity of scab resistance among related trees at local spatial scales. The genetics of resistance

Table 6. Heritability of pecan scab severity by family $\left(\mathrm{H}_{\mathrm{f}}{ }^{2}\right)$ and by tree $\left(\mathrm{H}_{\mathrm{t}}{ }^{2}\right)$ based on either scale data (1998), percentage area diseased (2013 and 2014), or scale data converted to midpoint data (1998) or percentage area diseased converted to scale data (2013 to 2014).

\begin{tabular}{llccccc}
\hline & & \multicolumn{2}{c}{ Family } & & \multicolumn{2}{c}{ Single tree } \\
\cline { 3 - 4 } Year & & $\mathbf{H}_{\mathbf{f}}^{\mathbf{2}}$ & $\mathbf{S E}^{\mathbf{z}}$ & & $\mathbf{H}_{\mathbf{t}}{ }^{2}$ & $\mathbf{S E}$ \\
\hline 1998 & Scale & 0.714 & 0.050 & & 0.202 & 0.040 \\
& \% area - midpoint & 0.703 & 0.053 & & 0.194 & 0.040 \\
2013 & Scale (converted) & 0.196 & 0.137 & & 0.036 & 0.031 \\
& \% area & 0.147 & 0.156 & & 0.026 & 0.032 \\
2014 & Scale (converted) & 0.323 & 0.109 & & 0.055 & 0.026 \\
& \% area & 0.069 & 0.136 & & 0.009 & 0.019 \\
\hline
\end{tabular}

${ }^{\mathrm{z}}$ Standard error.

Table 4. Correlation analysis ${ }^{\mathrm{z}}$ between severity of pecan scab in Byron, GA, in 1998, 2013, and 2014 and environmental variables (Table 1) from each provenance location

\begin{tabular}{lllllllll}
\hline Year & Latitude $\left({ }^{\circ}\right)$ & $\begin{array}{c}\text { Precipitation } \\
\left(\mathbf{c m ~ y e a r}^{-1}\right)\end{array}$ & Max temp $\left({ }^{\circ} \mathbf{C}\right)$ & Min temp $\left({ }^{\circ} \mathbf{C}\right)$ & Elevation $(\mathbf{m})$ & Bud break & Leaflet droop $\left({ }^{\circ}\right)$ & Leaflet tilt $\left({ }^{\circ}\right)$ \\
\hline $1998($ scale $)$ & $-0.693(0.001)$ & $-0.823(<0.0001)$ & $0.677(0.002)$ & $0.633(0.004)$ & $0.663(0.002)$ & $-0.560(0.01)$ & $-0.883(<0.0001)$ & $-0.844(<0.0001)$ \\
$1998(\%)$ & $-0.564(0.01)$ & $-0.868(<0.0001)$ & $0.524(0.02)$ & $0.566(0.01)$ & $0.426(0.07)$ & $-0.549(0.02)$ & $-0.803(<0.0001)$ & $-0.730(0.0004)$ \\
2013 & $-0.267(0.3)$ & $-0.716(0.0006)$ & $0.508(0.03)$ & $0.313(0.2)$ & $0.093(0.7)$ & $-0.413(0.08)$ & $-0.728(0.0004)$ & $-0.611(0.006)$ \\
2014 & $-0.187(0.4)$ & $-0.625(0.004)$ & $0.491(0.03)$ & $0.251(0.3)$ & $0.017(0.9)$ & $-0.371(0.1)$ & $-0.700(0.0009)$ & $-0.533(0.02)$ \\
\hline
\end{tabular}

${ }^{\mathrm{z}}$ With scale (category) data, Spearman's rank correlation was used. With continuous data (\%), Pearson's correlation was used. Values in parentheses are $P$-values.

Table 5. Stepwise multiple regression ${ }^{x}$ between scab severity on pecan trees from different provenances in three different years and provenance location environmental variables and pecan foliage characteristics

\begin{tabular}{|c|c|c|c|c|c|c|c|}
\hline \multirow[b]{2}{*}{ Year } & \multirow[b]{2}{*}{ Total $\mathbf{R}^{2}$} & \multicolumn{6}{|c|}{ Partial $R^{2}$ y (beta coefficient) $^{z}$} \\
\hline & & Precipitation & Elevation & Latitude & Minimum temp & Budbreak & Leaflet droop \\
\hline 1998 (scale) & 0.89 & $0.77(-0.63)$ & $\mathrm{ns}$ & $\mathrm{ns}$ & $\mathrm{ns}$ & $0.02(0.22)$ & $0.11(-0.56)$ \\
\hline $1998(\%)$ & 0.89 & $0.74(-0.86)$ & ns & ns & ns & $0.09(0.50)$ & $0.06(-0.52)$ \\
\hline 2013 & 0.86 & $0.12(-0.79)$ & $0.13(-0.47)$ & $\mathrm{ns}$ & $\mathrm{ns}$ & $0.08(0.49)$ & $0.53(-0.74)$ \\
\hline 2014 & 0.81 & $0.14(-0.61)$ & ns & $0.19(0.85)$ & $\mathrm{ns}$ & ns & $0.49(-0.85)$ \\
\hline
\end{tabular}

${ }^{\mathrm{x}}$ Results of ANOVA by year: 1998 (scale) model F-value $42.7=(P<0.0001), 1998(\%)$ model F-value $=37.2(P<0.0001), 2013$ model F-value $=22.0(P<$ $0.0001), 2014$ model F-value $=21.9(P<0.0001)$.

${ }^{\mathrm{y}}$ The partial $\mathrm{R}^{2}$ value describes the proportion of the variance accounted for by that predictor in the model.

${ }^{\mathrm{z}}$ The beta coefficient (also known as the standardized estimate) allows direct comparison of the relative strength of the various predictors within the model (the beta coefficient is measured in standard deviations, not the actual units of the variables, so they can be compared directly to one another). 
to scab in pecan is not understood, but diversity in susceptibility of progeny in families has been noted previously (Thompson and Grauke 1994). Heritability of scab resistance is considered to be sufficiently high to allow breeding to make reasonable progress (Thompson and Grauke 1994). The results of the current study on heritability were not consistent. In 1998, family heritability was 0.714 , indicating good heritability, and comparable to that found by Thompson and Grauke ( 0.500). However, in 2013 and 2014 (using the percentage scale), family heritability was rather low $(0.147$ and 0.069 , respectively), but improved slightly if the \% data were converted to the 1 to 5 rating scale ( 0.196 and 0.323 , respectively). Further work is needed to establish heritability of pecan scab. Using a 1 to 5 rating scale and basing that score on the maximum severity encountered in a canopy may offer some advantages in estimating heritability in this respect, as well as being a more rapid approach to disease assessment. However, these types of category scales have been shown to be associated with a greater risk of type II error (Bock et al. 2010; Chiang et al. 2014). The similarity in results among the three years shows that both methods were adequate to demonstrate differences among provenances and among families in provenances. Differences among years might, at least in part, be due to the different assessment methods used to compare trees, "escapes" from infection, prevailing weather conditions, the individual raters in different years, or changes in the pathogen population. Pecan leaves develop over a period of several weeks, and remain susceptible only during the period of leaf expansion (generally April through June, but for individual leaves this lasts only 10 to 14 days). Lack of sufficient inoculum during leaf production and development would reduce infection. Finally, the different individual raters involved could also be a source of variation between years; interrater variability is one of the sources of error in plant disease assessment (Bock et al. 2008; Nutter et al. 1993).

The study was conducted at a single location. Pathogenic specialization exists in F. effusum (Conner 2013; Conner and Stevenson 2004; Converse 1960; Demaree and Cole 1929), and it is known to be genetically diverse (Bock et al. 2014a, c). Thus, those trees resistant at Byron in 1998 and again 16 and 17 years later will not necessarily be resistant if exposed to the scab pathogen at other locations. The Byron location has 600 acres of pecan with numerous cultivars that are known to have varying susceptibility to scab (Thompson and Grauke 1994), ensuring that at least to some degree, these trees have been exposed to a wide range of pathogenic variants of $F$. effusum. But the risk of resistance failing at another location should be considered prior to utilizing any of the material identified herein.

The results of this study are consistent, but analysis based on an assessment of scab severity on the fruit in the same trees should be conducted to corroborate observations on foliage. Fortunately there is a good relationship between severity of scab on leaves and fruit (Goff et al. 2003; Nesbitt et al. 1996; Nutter et al. 1985; Sanderlin et al. 1981). However, not all trees have produced fruit, and some have severe alternate bearing, making a comprehensive collection problematic. As the trees get older, bearing may become more consistent on more trees.

The results of this study confirm the range in scab susceptibility that exists within the pecan metapopulation. Much of this variation is likely due to rainfall at the source location for the genotype, which drives scab epidemics. This genetic variation in regard to scab susceptibility is important to characterize as it is a basis for selection of material likely to have favorable traits conferring scab resistance. Thus certain populations or source areas of pecan are more likely to have scab resistance compared with others as a result of selection where rainfall and other local conditions are conducive to regular scab epidemics. Those materials most likely to contribute to genetic improvement regarding scab resistance are identified in the provenance collection.

\section{Acknowledgments}

We acknowledge the time and input of Shirley Anderson, Jacob Werner, and Kaylee Carson, who helped with sample collection, assessment, and data input. We also appreciate the contributions of Dr. Charles 'Chuck' Riley for his work on the 1998 collections and insights into the provenance collection in relation to scab. We appreciate the funding support of the USDA-ARS through CRIS project 6042-21220-012-00. This article reports the results of research only. Mention of a trademark or proprietary product is solely for the purpose of providing specific information and does not constitute a guarantee or warranty of the product by the U.S. Department of Agriculture and does not imply its approval to the exclusion of other products that may also be suitable.

\section{Literature Cited}

Beedanagari, S. R., Dove, S. K., Wood, B. W., and Conner, P. J. 2005. A first linkage map of pecan cultivars based on RAPD and AFLP markers. TAG Theor. Appl. Genet. 110:1127-1137.

Bock, C. H., Cottrell, T. E., Hotchkiss, M. W., and Wood, B. W. 2013. Vertical distribution of scab in large pecan trees. Plant Dis. 97:626-634.

Bock, C. H., Endalew, T. T., Biswas, B. K., Yadav, A. K., Sitther, V., Hotchkiss, M. W., Stevenson, K. L., and Wood, B. W. 2014a. A comparison of UP-PCR and RAPD markers to study genetic diversity of the pecan scab fungus (Fusicladium effusum G. Winter). For. Pathol. 44:266-275.

Bock, C. H., Gottwald, T. R., Parker, P. E., Ferrandino, F., Welham, S., van den Bosch, F., and Parnell, S. 2010. Some consequences of using the HorsfallBarratt scale for hypothesis testing. Phytopathology 100:1030-1041.

Bock, C. H., Hotchkiss, M. W., Cottrell, T. E., and Wood, B. W. 2015. The effect of sample height on spray coverage in mature pecan trees. Plant Dis. 99:916-925.

Bock, C. H., Hotchkiss, M. W., Brenneman, T. B., Stevenson, K. L., Goff, W., Smith, M. W., Wells, L., and Wood, B. W. 2014b. Hedging - does it affect the severity of pecan scab? Pecan Grower 25:48-58.

Bock, C. H., Parker, P. E., Cook, A. Z., and Gottwald, T. R. 2008. Visual rating and the use of image analysis for assessing different symptoms of citrus canker on grapefruit leaves. Plant Dis. 92:530-541.

Bock, C. H., Stevenson, K. L., Arias, R. S., and Wood, B. W. 2014c. Genetic diversity and population structure of Fusicladium effusum on pecan in the U.S.A. Plant Dis. 98:916-923.

Campbell, C. L., and Madden, L. V. 1990. Monitoring epidemics: Environment Pages 43-73 in: Introduction to Plant Disease Epidemiology. Wiley, London.

Chiang, K.-S., Liu, S.-C., Bock, C. H., and Gottwald, T. R. 2014. What interval characteristics make a good categorical disease assessment scale? Phytopathology 104:575-585.

Cole, J. R., and Gossard, A. C. 1956. Increased virulence of scab (Cladosporium effusum Wint. Demaree) on Stuart pecan in Mississippi and its presence in Louisiana. Plant Dis. Rep. 40:1120.

Conner, P. J. 2013. Evaluation of response to pecan scab pathogen race using a detached leaflet protocol. HortScience 48:63-67.

Conner, P. J., and Stevenson, K. L. 2004. Pathogenic variation of Cladosporium caryigenum isolates and corresponding differential resistance in pecan HortScience 39:553-557.

Conner, P. J., and Wood, B. W. 2001. Identification of pecan cultivars and their genetic relatedness as determined by randomly amplified polymorphic DNA analysis. J. Am. Soc. Hortic. Sci. 126:474-480.

Converse, R. H. 1960. Physiologic specialization in Fusicladium effusum and its evaluation in vitro. Phytopathology 50:527-531.

Cooper, J. N., and Johnson, J. D. 1985. The influence of tree spacing and fungicide use on the susceptibility of 'Western' to pecan scab. Pages 37-38 in: Proceedings of the $63^{\text {rd }}$ and $64^{\text {th }}$ Texas Pecan Growers Association Annual Conferences, San Antonio, Texas, 8-11 July, 1984 and Galveston, Texas, 7-10 July, 1985.

Demaree, J. B., and Cole, J. R. 1929. Behavior of Cladosporium effusum (Wint.) Demaree on some varieties of pecan. J. Agric. Res. 38:363-370.

Erdin, N., Tartarini, S., Broggini, G. A. L., Sansavini, S., Gessler, C., and Patocchi, A. 2006. Mapping the apple scab-resistance gene $V b$. Genome 49:1238-1245.

Goff, W. D., McVay, J. R., and Gazaway, W. S. 1996. Page 222 in: Pecan production in the Southeast. Alabama Cooperative Extension System Circular ANR-459. Auburn University, Auburn, AL.

Goff, W. D., Nesbitt, M. L., and Browne, C. L. 2003. Incidence of scab and foliage condition on pecan cultivars grown without fungicide or insecticide sprays in a humid region. HortTech. 13:381-384.

Gottwald, T. R. 1985. Influence of temperature, leaf wetness period, leaf age, and spore concentration on infection of pecan leaves by conidia of Cladosporium caryigenum. Phytopathology 75:190-194.

Gottwald, T. R., and Bertrand, P. F. 1982. Patterns of diurnal and seasonal airborne spore concentrations of Fusicladium effusum and its impact on a pecan scab epidemic. Phytopath. 72:330-335.

Gottwald, T. R., and Bertrand, P. F. 1983. Effect of time of inoculation with Cladosporium caryigenum on pecan scab development and nut quality. Phytopath. 73:714-718.

Gottwald, T. R., and Bertrand, P. F. 1988. Effects of an abbreviated pecan disease control program on pecan scab disease increase and crop yield. Plant Dis. 72:27-32.

Grauke, L. J., Iqbal, M. J., Reddy, A. S., and Thompson, T. E. 2003a. Development of microsatellite DNA markers in pecan. J. Am. Soc. Hortic. Sci. 128:374-380.

Grauke, L. J., Mendoza-Herrera, M., Miller, A., and Wood, B. W. 2011. Geographic patterns of genetic variation in native pecans. Tree Gen. Geno. 7:917-932.

Grauke, L. J., Payne, J. A., and Wood, B. W. 1989. North American pecans: A provenance study. Annu. Rep. N. Nut Growers Assoc. 80:124-131. 
Grauke, L. J., Storey, J. B., Thompson, T. E., and Wood, B. W. 2003b. Leaf structure and nutrient content vary in native pecan populations. Proc. Tex. Pecan Growers Assoc. 70:59-60.

Grauke, L. J., Thompson, T. E., and Marquard, R. D. 1995. Evaluation of pecan [Carya illinoinensis (Wangenh.) K. Koch] germplasm collections and designation of a core subset. HortSci. 30:950-954.

Holland, J. B., Uhr, D. V., Jeffers, D., and Goodman, M. M. 1998. Inheritance of resistance to southern corn rust in tropical-by-corn-belt maize populations. Theor. Appl. Genet. 96:232-241.

Hunter, R. E., and Roberts, D. D. 1978. A disease grading system for pecan scab. Pecan Q. 12:3-6.

Lamari, L. 2008. ASSESS 2.0: Image Ana lysis Software for Plant Disease quantification. APS Press, St. Paul, MN.

Latham, A. J. 1982. Effects of some weather factors and Fusicladium effusum conidium dispersal in pecan scab occurrence. Phytopathology 72:1339-1345.

Nesbitt, M., Goff, W. D., Hale, T., Thompson, T., Reynolds, K. L., and O'barr, R. D. 1996. Screening 147 pecan cultivars for scab resistance. Proc. Se Pecan Growers Assoc. 89:90-97.

Nutter, F. W., Jr., Gleason, M. L., Jenco, J. H., and Christians, N. L. 1993. Accuracy, intra-rater repeatability, and inter-rater reliability of disease assessment systems. Phytopathology 83:806-812.

Nutter, F. W., Jr., Gottwald, T. R., and Bertrand, P. F. 1985. Relationship between pecan scab disease progression on leaves versus nuts in three pecan cultivars. Phytopathology 75:1369 (abstract 722).

Rüter, B., Hamrick, J. L., and Wood, B. W. 1999. Genetic diversity within provenance and cultivar germplasm collections versus natural populations of pecan (Carya illinoinensis). J. Hered. 90:521-528.

Sagaram, M., Lombardini, L., and Grauke, L. J. 2011. Variation in anatomical characteristics in leaves of pecan seedstocks from Mexico and the United States. J. Am. Soc. Hortic. Sci. 136:103-108.

Sanderlin, R. S. 1995. Effect of nut scab on pecan yield and quality components. Pages 45-49 in: Sustaining Pecan Productivity into the 21st Century: Second National Pecan Workshop Proceedings, Wagoner, Oklahoma, 23-26 July, 1994. U.S. Dept. Agric., Agriculture Research Service.

Sanderlin, R. S., Hunt, A. S., and Young, W. A. 1981. Reaction of pecan varieties to scab disease in Louisiana. Pecan South 8:6-9.
Saxton, A. M. 1998. A macro for converting mean separation output to letter groupings in PROC MIXED. Pages 1243-1246 in: Proc. 23rd SAS Users Group Intl., Nashville, TN, 22-25 March. (http://www2.sas.com/proceedings/sugi23/Stats/p230.pdf).

Seyran, M., Brenneman, T. B., and Stevenson, K. L. 2010. A rapid method to monitor fungicide sensitivity in the pecan scab pathogen, Fusicladium effusum. Crop Prot. 29:1257-1263.

Sparks, D., Yates, I. E., Bertrand, P. F., and Brenneman, T. B. 2009. The relative importance of elevation and rainy days on the incidence of scab damage of pecan nuts in the southeastern USA. J. Hortic. Sci. Biotechnol. 84:137-142.

Stevenson, K. L. 1999. Fungicide resistance management in pecans. South East Pecan Growers Association 92:58-64.

Stevenson, K. L., and Bertrand, P. F. 2001. Within-season dynamics of yield loss due to pecan scab fruit infections. Phytopathology 91:S85.

Stevenson, K. L., Bertrand, P. F., and Brenneman, T. B. 2004. Evidence for reduced sensitivity to propiconazole in the pecan scab fungus in Georgia. Phytopathology 94:S99.

Thompson, T. E., and Grauke, L. J. 1991. Pecans and hickories (Carya). Pages 839-904 in: Genetic Resources of Temperate Fruit and Nut Crops. J. N. Moore and J. R. Ballington, eds. International Society for Horticultural Science, Wageningen, Netherlands.

Thompson, T. E., and Grauke, L. J. 1994. Genetic resistance to scab disease in pecan. HortScience 29:1078-1080

Wells, L., Brenneman, T., Brock, J., Culpepper, A. S., Hudson, W., and Mitchem, W. 2015. 2015 Commercial pecan spray guide. University of Georgia Extension. Bulletin 841. http://www.caes.uga.edu/commodities/fruits/pecan/documents/ 2015-Pecan-Spray-Guide.pdf

Wood, B. W., Grauke, L. J., and Payne, J. A. 1998. Provenance variation in pecan J. Am. Soc. Hortic. Sci. 123:1023-1028.

Wright, J. W. 1976. Provenance testing. Pages 253-265 in: Introduction to Forest Genetics. Academic Press, New York.

Xu, X.-M., Roberts, T., Barbara, D., Harvey, N. G., Gao, L.-Q., and Sargent, D. J. 2009. A genetic linkage map of Venturia inaequalis, the causal agent of apple scab. BMC Res. Notes 2:163.

Yadav, N. V. S., de Vos, S. M., Bock, C. H., and Wood, B. W. 2013. Development and validation of standard area diagrams to aid assessment of pecan scab symptoms on fruit. Plant Pathol. 62:325-335. 